\title{
Management of radiation dose reduction in computed tomography: an experience at burapha university hospital, thailand
}

\author{
Limchareon $\mathrm{S}^{*}$, Kaowises K and Saensawas W \\ Division of Radiology and Nuclear Medicine, Faculty of Medicine, Burapha University, Thailand
}

\begin{abstract}
Objective: The purpose of this study was to quantify the dose reduction for each computed tomography (CT) reduction dose protocol.

Subject and Method: One hundred and fifty repeat CT examinations with an implementation of a CT dose reduction technique per examination were included. Percent of dose reductions compared with their prior CT examinations with standard CT protocols in terms of dose length product were calculated.

Result: There were 95 examinations using scanning parameter optimization technique, 47 examinations using reduction of multiphase scanning and 8 examinations using limited scan range. Percent of dose reductions for scanning parameter optimization, reduction of multiphase scanning and limitation of scan range were $43.3 \%$, $52.5 \%$, and $39.1 \%$ respectively. Among the scanning parameter optimization, percent of dose reductions by lowering tube voltage, and lowering tube current were $47.9 \%$, and $38.6 \%$, respectively.

Conclusion: The presented dose lowering protocols are simple and available in most modern CT scanners. Reduction of multiphase scanning shows highest dose reduction. All of these techniques do not depend on CT scanners but requiring special CT protocol tailored for the individual patient for optimal diagnostic quality in the optimization of patient dose in CT.
\end{abstract}

Abbreviations: ACR - American college of radiology; CT computed tomography; DLP - dose length product; $\mathrm{kVp}$ - kilovotage peak; $\mathrm{mA}$ - milliampere; MDCT - multi-detector computerized tomography; IR - iterative reconstruction

\section{Introduction}

Since the era of multi-detector computerized tomography (MDCT), the rate of computed tomography (CT) examinations in our institution has been increasing rapidly, similar to another part of the world [1]. Although MDCT has a lot of benefits, the radiation dose from MDCT is higher than other imaging modalities [2]. Thus, the risk of carcinogenesis from radiation is to be concerned [2]. A retrospective study in a large group of patients published in 2012 showed significant associations between radiation doses from CT scans and subsequent incidence of leukemia and brain tumors in children aged younger than 15 years [3]. But awareness of this possible risk is less concerned among physician as demonstrated by a survey in emergency department (ED) physicians that, only $9 \%$ of them believed that radiation increases cancer risk [4]. Even radiologists in that study, less than $50 \%$ of them believed that radiation increases cancer risk and most of them were unable to correctly estimate the dose for one CT scan compared with a chest x-ray [4]. Similarly, most ED physicians and medical students were unable to correctly estimate the dose for one CT scan compared with a chest $\mathrm{x}$-ray [4-5]. In addition, almost $80 \%$ of medical students and interns who are future physicians have never heard of the American College of Radiology (ACR) appropriateness criteria [5-6].

Many efforts have tried to minimizing radiation dose from CT either by vendors or users. All vendors have available dose reduction technology and some of the technologies are being in research. Whereas CT users can minimize radiation dose by scanning parameter optimization, reduction of inappropriate multiphase scanning, limiting the scan range in the $\mathrm{Z}$-axis, and applying innovative technologies such as iterative reconstruction (IR) algorithms that are available in some new CT scanners.

Our institution has installed a 64-slice MDCT (Toshiba Aquilian 64; Otawara, Japan) since April 2013. Initially, we used standard protocols for all of CT examinations. After a period of time, we have noticed that there were a number of follow-up cases in our institution, and rapidly increasing as shown in figure 1. Then CT protocols for reducing radiation dose in such cases have been implemented.

The aim of this study was to measure and express the amount of dose reduction in different $\mathrm{CT}$ techniques. We compared low-dose CT protocol with standard-dose CT protocol in the same patient. We do not aim to compare the statistically significant difference between these techniques, because all of these techniques should be used, even in the single examination.

Correspondence to: Limchareon S, Division of Radiology and Nuclear Medicine, Faculty of Medicine, Burapha University, Thailand, Tel: +6638386554; Fax: +6638386557; E-mail: sornsupha@hotmail.com

Key words: computed tomography, dose reduction, radiation dose

Received: January 02, 2018; Accepted: January 19, 2018; Published: January 23, 2018 


\section{Number of follow-up cases 2013-2016}

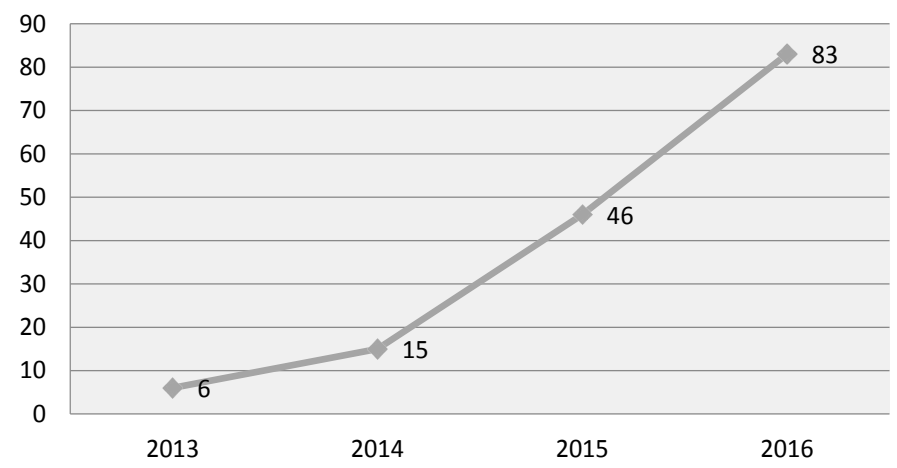

Figure 1. Number of follow-up cases at Burapha University hospital.

\section{Material and method}

This study was retrospectively designed in a 160-bed university hospital. The university institutional review board approved this study, No.17/2560. Informed consent was waived due to retrospective nature of the study.

We searched the CT database for all patients who underwent repeat $\mathrm{CT}$ examinations and received dose-lowering protocols in the follow-up examinations since the CT installation on April 2013 to December 2016. A total of 150 CT examinations were identified. The CT procedures were performed for the clinical indications of followup in patients as illustrated in table 1 . Radiation dose estimates were determined using the dose-length product (DLP) in mGy-cm. The DLP is a measurement of the radiation delivered by a CT scanner for each examination multiplied by scan length [7]. The DLP which is displayed on the scanner console was used for comparing radiation dose per examination in a single patient. Each repeat examination was obtained at almost the identical anatomic location to the prior examination. The SPSS version 22 was used for statistical analysis. All results are reported as frequency, percent, mean and standard deviation as appropriate. Paired t-test was used to compare the radiation doses between the first and the second examinations.

\section{CT dose lowering protocols}

The initial CT examinations were performed using standard CT technical scanner settings (helical mode and fixed tube potential at 120 kilovoltage peak $[\mathrm{kVp}])$. The head and neck $\mathrm{CT}$ examinations were performed using fixed tube current at 300 milliamperes $(\mathrm{mA}), 0.75$ second (s) rotation time, pitch 0.8 and $2 \mathrm{~mm}$ reconstruction interval. The chest, abdomen and KUB (Kidney-Ureter-Bladder) CT were done, using tube current modulation [8] 275-500 mA, 0.5 s rotation time, pitch 0.8 and $2 \mathrm{~mm}$ reconstruction interval. The dose lowering techniques included; 1) optimized technical scanner settings, 2) limited image creep, and 3) limited phase creep. Of the optimized technical scanner settings, we subdivided into lowering tube current and lowering tube potential. Among lowering tube current, we reduced the tube current to $225 \mathrm{~mA}$, in cases of fixed tube current whilst in cases of using tube current modulation, we limited the maximum range of tube current to $400 \mathrm{~mA}$. In cases of lowering tube potential, we reduced to $100 \mathrm{kVp}$ and fixed tube current at $250 \mathrm{~mA}$. Of the limited image creep, we limited image creep to only the region of interest, either on non-contrast phase or on delayed phase. Similarly, in the limited phase creep, we omitted one, two or three phases, usually a non-contrast phase, and/or latearterial phase, and/or delayed phase. In a single patient, we used only one technique per one examination whereas the other parameters were held the same as the prior examination.

\section{Result}

The study included 150 patients, mean age 53.2 years (range, 13 years to 88 years). Of whom, 62 were female ( $41.3 \%$ ) and 88 were male (58.7\%). The number of patients, mean difference and percentage of dose reduction values with respect to dose-lowering techniques are presented in table 2 . The highest dose reduction technique was for the limited phase creep (average 52.5\%), while the lowest dose reduction technique was for the decreased tube current (38.6\%).

\section{Discussion}

Although current evidence is sufficient that, radiation increases the risk of cancer [2-3], benefits from CT in timely and correct diagnosis have increased the usefulness of CT [1]. CT radiation doses depend on patient size, scan coverage, imaging parameters, and CT scanner technology. The current study demonstrates that the limited scan phase had the highest dose reduction (52.5\%). The unindicated multiphase scans are usually overlooked by staff, according to the study by Guite et al [9]. They studied abdominal and pelvic CT examinations and reported that $52.8 \%$ of phases were not supported by ACR appropriateness criteria and $35.8 \%$ of phases were un-indicated. Limited scan phase technique is very simple, no image quality penalty and most importantly, this technique does not depend on CT scanners.

Limited image creep is one of the simplest techniques for

Table 1. Indications for follow up.

\begin{tabular}{|c|c|c|}
\hline Dose lowering techniques & Indications & No. of patients \\
\hline \multicolumn{3}{|c|}{$\begin{array}{l}\text { 1.Optimized technical scanner } \\
\text { settings }\end{array}$} \\
\hline \multirow[t]{3}{*}{ 1.1 Decreased tube current } & Intracranial hematoma/infarct/mass & 72 \\
\hline & Urinary tract stone/cyst & 8 \\
\hline & Abdominal cancer & 1 \\
\hline \multirow[t]{3}{*}{$\begin{array}{l}\text { 1.2 Decreased tube } \\
\text { potential }\end{array}$} & Urinary tract stone & 12 \\
\hline & $\begin{array}{l}\text { Post-abdominal aortic aneurysm } \\
\text { stent }\end{array}$ & 1 \\
\hline & Pneumomediastinum & 1 \\
\hline \multirow[t]{4}{*}{ 2.Limited image creep } & Abdominal cancer & 3 \\
\hline & Renal nodule/cyst & 2 \\
\hline & Intra-abdominal collections & 2 \\
\hline & C-spine fracture & 1 \\
\hline \multicolumn{3}{|l|}{ 3.Limited phase creep } \\
\hline \multirow[t]{6}{*}{3.1 omitted 1 phase } & Abdominal cancer & 22 \\
\hline & Liver nodule/cyst & 2 \\
\hline & Hepatobiliary infection & 2 \\
\hline & Intra-abdominal collections & 1 \\
\hline & Intra-abdominal foreign body & 1 \\
\hline & Kidney injury & 1 \\
\hline \multirow[t]{7}{*}{3.2 omitted 2 phases } & Abdominal cancer & 5 \\
\hline & Liver nodule/cyst & 3 \\
\hline & Kidney injury & 1 \\
\hline & Post-operative pain & 1 \\
\hline & Intra-abdominal collections & 1 \\
\hline & Adrenal gland nodule & 1 \\
\hline & Pulmonary tuberculosis & 1 \\
\hline \multirow[t]{4}{*}{3.3 omitted 3 phases } & Abdominal cancer & 2 \\
\hline & Adrenal gland nodule & 2 \\
\hline & Intra-abdominal infection & 1 \\
\hline & & 150 \\
\hline
\end{tabular}


Table 2. Mean difference and percentage of dose reduction.

\begin{tabular}{|c|c|c|c|c|}
\hline $\begin{array}{l}\text { Dose lowering technique } \\
\text { (n) }\end{array}$ & Mean difference (SD) & $95 \%$ Confidence interval & Dose reduction (\%) & $p$ \\
\hline \multicolumn{5}{|l|}{ 1.Optimized technical scanner settings } \\
\hline 1.1 decreased tube current $(81)$ & $526.6(470.1)$ & $422.7-630.6$ & 38.6 & $<0.001^{*}$ \\
\hline 1.2 decreased tube potential (14) & $643.8(404.8)$ & $409.4-876.9$ & 47.9 & $<0.001^{*}$ \\
\hline 2.Limited image creep (8) & $1152.9(413.1)$ & $807.5-1498.3$ & 39.1 & $<0.001 *$ \\
\hline \multicolumn{5}{|l|}{ 3.Limited phase creep } \\
\hline 3.1 omitted 1 phase $(29)$ & $1295.9(721.6)$ & $1021.4-1570.4$ & 36.7 & $<0.001^{*}$ \\
\hline 3.2 omitted 2 phases (13) & $2767.2(1437.0)$ & $1898.8-3635.5$ & 58.7 & $<0.001^{*}$ \\
\hline 3.3 omitted 3 phases $(5)$ & $3103.9(1779.2)$ & $894.7-5313.0$ & 62.1 & $0.018 *$ \\
\hline
\end{tabular}

$\mathrm{n}=$ number of examinations, $\mathrm{SD}=$ Standard deviation, $*=$ Statistical significance

minimizing radiation, especially in follow-up patients. The patients are previously diagnosed and the region of interest that is required is specifically scanned. Individualize CT scans according to the region of interest by the radiologist is mandated.

For the optimized technical scanner settings included; lowering tube current, lowering tube potential, and increased pitch, all of these techniques result in image deterioration by increasing noise [8]. Therefore, image quality and exposure dose reduction are a tradeoff. Punwani et al. [10] studied in phantoms for pulmonary nodule detection. They demonstrated that the sensitivity and specificity for pulmonary nodule detection in phantoms decreased when the noise increased. As shown by the study in pulmonary embolism that, the simulated reduction in CT doses had a significant adverse effect on the readers' diagnostic confidence and image quality [11]. However, in clinical practices, authors achieved an excellent diagnostic image quality with a low dose and ultra-low-dose techniques in the diagnosis of infectious lung disease [12] as well as an excellent sensitivity (97\%) and specificity (96\%) for diagnosing renal colic [13]. A recent article with a large number of patients and various clinical settings, they showed significantly lower CT radiation doses to 53\%-73\% while maintained acceptable diagnostic image quality [14]. Though in that study, the groups of patients between before and after dose-reduction implementation were not the same and two reduction techniques were used at the same time. Previous literature has proposed a reconstruction algorithm to provide diagnostic quality images in minimizing radiation dose settings [15]. They demonstrated the benefits of resampling the scan data by resampling $5 \mathrm{~mm}$ thick slice acquisitions to $0.6 \mathrm{~mm}$ slices and then producing $2 \mathrm{~mm}$ coronal MPRs, that improved the spatial resolution but with a moderate noise penalty. Recently, objective data have shown that the use IR significantly reduced noise, compared to no IR with the other identical parameters $(p<0.017)$ [16]. However, IR is innovative technology and is not available in our CT scanner.

The tube current $(\mathrm{mA})$ modulation is the dose reduction technique which is usually available in the modern MDCT scanners and already set in the standard CT protocols [8]. The scan $\mathrm{mA}$ value is adjusted, along with the length of the patient, for each tube rotation. The $\mathrm{mA}$ modulation technique has been reported to reduce $40 \%$ of radiation dose per examination [8]. In the current study, we limited the maximum range of the variable $\mathrm{mA}$. As a result, we can further reduce the radiation dose to $38.6 \%$. Decreasing tube current has a direct linear effect on the decreasing radiation does whereas the radiation dose does not decrease proportionally to the tube potential [17]. We decreased tube potential from $120 \mathrm{kVp}$ to $100 \mathrm{kVp}$ and the result showed $47.9 \%$ of dose reduction. However, decreased tube potential is not recommended in the large patient because beam attenuation increases exponentially with increasing patient thickness [18]. Recently, there has been automatic tube potential selection technique in some vendors [17].
Radiologist's bias is the major limitation of our study. The doselowering CT protocols were initiated by an author (L.S) who interpreted the CT images. In addition, these CT examinations were follow-up examinations and the radiologist were aware of the previous results. This may not be generalized to the other clinical indications. We did not try to compare the image quality or accuracy which is an important effect on the diagnostic outcome. Further assessment of the accuracy of each technique is required. Some dose-lowering protocols have considerable image noise [19], therefore the usability may be limited to some diagnostic questions. The DLP that we used to represent the radiation dose does not the true patient effective dose. Lastly, a small number of patients often limited the study results.

In conclusion, we present ready-to-use types of radiation doselowering techniques. These techniques are simple and likely available in most CT scanners. The limited phase creep technique reduces highest radiation dose whereas the reduced tube current is the least. We would like to emphasize on radiologists' awareness and promote all of these techniques to be used in daily practice especially in patients who receive repeat imaging. Nevertheless, these techniques require a special protocol tailored for the individual patient and thus need focus and change in the radiologists' practices.

\section{Acknowledgement}

The authors are grateful to Faculty of Medicine, Burapha University for all supports.

\section{Conflict of interest}

No conflict of interest to disclose.

\section{References}

1. Fletcher JG, Kofler JM, Coburn JA, Bruining DH, McCollough CH (2013) Perspective on radiation risk in CT imaging. Abdom Imaging 38: 22-31. [Crossref]

2. Sodickson A, Baeyens PF, Andriole KP, Prevedello LM, Nawfel RD, et al. (2009) Recurrent CT, cumulative radiation exposure, and associated radiation-induced cancer risks from CT of adults. Radiology 251: 175-184. [Crossref]

3. Pearce MS, Salotti JA, Little MP, McHugh K, Lee C, et al. (2012) Radiation exposure from $\mathrm{CT}$ scans in childhood and subsequent risk of leukaemia and brain tumours: a retrospective cohort study. Lancet 380: 499-505. [Crossref]

4. Lee CI, Haims AH, Monico EP, Brink JA, Forman HP (2004) Diagnostic CT scans: assessment of patient, physician, and radiologist awareness of radiation dose and possible risks. Radiology 231: 393-398. [Crossref]

5. Prezzia C, Vorona G, Greenspan R (2013) Fourth-year medical student opinions and basic knowledge regarding the field of radiology. Acad Radiol 20: 272-283. [Crossref]

6. Saha A, Roland RA, Hartman MS, Daffner RH (2103) Radiology medical student education: an outcome-based survey of PGY-1 residents. Acad Radiol 20: 284-289. [Crossref]

7. Goldman LW (2007) Principles of CT: radiation dose and image quality. J Nucl Med Technol 35: 213-225. [Crossref] 
8. Mayo-Smith WW, Hara AK, Mahesh M, Sahani DV, Pavlicek W (2014) How I do it: managing radiation dose in CT. Radiology 273: 657-672. [Crossref]

9. Guite KM, Hinshaw JL, Ranallo FN, Lindstrom MJ, Lee Jr FT (2011) Ionizing radiation in abdominal CT: unindicated multiphase scans are an important source of medically unnecessary exposure. J Am Coll Radiol 8: 756-761. [Crossref]

10. Punwani S, Zhang J, Davies W, Greenhalgh R, Humphries P (2008) Paediatric CT: the effects of increasing image noise on pulmonary nodule detection. Pediatr Radiol 38: 192-201. [Crossref]

11. MacKenzie JD, Nazario-Larrieu J, Cai T, Ledbetter MS, Duran-Mendicuti MA, et al. (2007) Reduced-dose CT: effect on reader evaluation in detection of pulmonary embolism. AJR Am Roentgenol Radiol 189: 1371-1379. [Crossref]

12. Rizzi EB, Schinina V, Gentile FP, Bibbolino C (2007) Reduced computed tomography radiation dose in HIV-related pneumonia: effect on diagnostic image quality. Clin Imaging 31: 178-184. [Crossref]

13. Poletti PA, Platon A, Rutschmann OT, Schmidlin FR, Iselin CE, et al. (2007) Low-dose versus standard-dose $\mathrm{CT}$ protocol in patients with clinically suspected renal colic. AJR Am Roentgenol Radiol 188: 927-933. [Crossref]
14. Sulagaesuan C, Saksobhavivat N, Asavaphatiboon S, Kaewlai R (2016) Reducing emergency $\mathrm{CT}$ radiation doses with simple techniques: a quality initiative project. $J$ Med Imaging Radiat Oncol 60: 23-34. [Crossref]

15. Ford NL, Protik A, Babyn P, Thomas K (2014) Does resampled image data offer quantitative image quality benefit for pediatric CT? J Biomed Sci Eng 7: 343-350.

16. Higashigaito K, Becker AS, Sprengel K, Simmen HP, Wanner G, et al. (2016) Automatic radiation dose monitoring for $\mathrm{CT}$ of trauma patients with different protocols: feasibility and accuracy. Clin Radiol 71: 905-911. [Crossref]

17. Lira D, Padole A, Kalra MK, Singh S (2015) Tube potential and CT radiation dose optimization. AJR Am Roentgenol Radiol 204: W4-W10. [Crossref]

18. Moser JB, Sheard SL, Edyvean S, Vlahos I (2017) Radiation dose-reduction strategies in thoracic CT. Clin Radiol 72: 407-420. [Crossref]

19. Hojreh A, Czerny C, Kainberger F (2005) Dose classification scheme for computed tomography of the paranasal sinuses. Eur J Radiol 56: 31-37. [Crossref]

Copyright: $(\mathbb{2} 2018$ Limchareon S. This is an open-access article distributed under the terms of the Creative Commons Attribution License, which permits unrestricted use, distribution, and reproduction in any medium, provided the original author and source are credited. 